\title{
Diabetes: a hospital perspective
}

\author{
Rowan Hillson MBE
}

Diabetes affects $7 \%$ of adults in the UK. Among hospital inpatients it is much more common. Adult hospital bed occupancy for people with diabetes varies with population and case mix. Percentages are often in the mid-teens and may exceed $20 \%{ }^{1}$ Length of stay (LoS) for people with diabetes greatly exceeds that of non-diabetic patients as do emergency readmissions. Day case admissions are fewer. (Please see the National Diabetes Information Service website for details. ${ }^{2}$ ) Many admissions are preventable. Patient and healthcare professional education with specialist diabetes team support reduces emergencies and prevents escalation if any occur. ${ }^{3}$

People with diabetes report bad experiences in hospital (Box 1). ${ }^{4}$ People who manage their own condition find themselves forbidden to do so. Most illnesses or operations affect glucose balance, as can drugs and fasting. Hospital diabetes care is challenging, requiring expert specialist care. Yet patients are often managed by doctors and nurses with little knowledge of diabetes or awareness of the dangers. Patients, if not confused or too unwell, are usually better than staff at controlling their diabetes in hospital and should be allowed to. The National Patient Safety Agency has issued a mandatory alert 'Safer administration of insulin.' It states 'a training programme should be put in place for all healthcare staff...expected to prescribe, prepare and administer insulin.' See the NHS Diabetes training site. ${ }^{5}$

'People with diabetes in hospital need the right expertise at the right time from a trained ward team supported by the diabetes specialist team.' Diabetic inpatient specialist nurses reduce admissions from accident and emergency, reduce clinical incidents, shorten LoS and improve patient experience. ${ }^{3}$ Every acute hospital should have prompt access to a diabetes inpatient specialist team with dedicated inpatient sessions and access to all diabetic inpatients on all units. The Think Glucose programme to improve the care of people with diabetes in hospital, is available from the NHS Institute for Innovation and Improvement. ${ }^{6}$

People with diabetes are at risk of a wide range of complications in hospital many of which can be reduced by meticulous care (Box 2). ${ }^{7}$ On admission, or preoperatively check for complications, for example heart, kidney, neuropathy, foot, skin, eyes, infection. Promptly refer diabetic foot problems to the specialist diabetic foot team (see Putting Feet First). ${ }^{8}$

Glucose control is important. Higher glucose levels (on admission or perioperatively) are associated with more complications. An early study showed benefits from intensive glucose control in critically ill patients but meta-analysis showed that this increased the risk of mortality and hypoglycaemia. ${ }^{9}$ Until clearer evidence is

Rowan Hillson MBE, national clinical director for diabetes
Box 1. Comments from people with diabetes. Reproduced from the Diabetes UK survey. ${ }^{4}$

'The meals he received in hospital were totally inadequate in terms of portion and balance.'

'...had to suffer avoidably inappropriate timing and amounts of insulin dosage, which were also inappropriate in relation to timing and amount of her food intake.'

'I had a hypo, the nurse was called and did not know what to do... it was left for another patient on my ward to give me a sugary drink and biscuits.'

'I am sure that none of the professionals I encountered on that visit had any experience of diabetes and no credence was given to my expertise and knowledge of my own condition. I am dreading having to go into hospital again ...'

'I hate having to go into hospital because of the disruption it causes to my diabetes. I worry about it when I go in and get very concerned, anxious and frustrated.'

Box 2. Risks for people with diabetes in hospital. Adapted from Reference 7 .

- Infection (eg urinary tract, wound, cannula/line/catheter site)

- Severe, long-lasting infections

- Antibiotic-resistant infections

- Hospital-acquired infections

- Poor wound healing, fistula formation

- Pressure sores

- Glycaemic instability (hypoglycaemia, ketoacidosis)

- Hypoglycaemic drug errors (eg insulin)

- Venous thrombosis

- Ischaemic events - myocardial, peripheral vascular, cerebral

- Metabolic complexity

- Renal failure

- Cardiac failure

- Less likely to return to their own home

- Death

- Increased resource use

available the American Diabetes Association 2010 guidance $^{10}$ is helpful: start insulin in critically ill patients with persistent glucose levels $>10 \mathrm{mmol} / \mathrm{l}$ aiming for 7.8-10 mmol/l. For noncritically ill patients aim for pre-meal glucose of 4-7.8 $\mathrm{mmol} / \mathrm{l}$ and a random level of 4-10 mmol/l. Avoid hypoglycaemia at all costs.

Other important measures are:

- ensure appropriate nutrition. Seek dieticians' advice

- check feet and heels daily. Use pressure prevention measures ${ }^{8}$ 
- wash hands

- insert cannulae cleanly. Check daily

- use dressing packs and gloves to view ulcers/wounds. Debride and dress them properly

- use the right antibiotic for appropriate duration.

- avoid methicillin-resistant Staphylococcus aureus or Clostridium difficile.

In summary, hospitals should have an inpatient diabetes specialist team, train all staff about diabetes, monitor and reduce insulin errors and ensure that glucose control and complications are checked on admission and throughout. Poor inpatient diabetes care is distressing, disabling and costly to all concerned. Much can be done to improve it.

\section{References}

1 Rayman G. National Diabetes Inpatient Audit Day 2009. Oral presentation Diabetes UK APC 2010. Unpublished.

2 National Diabetes Information Service. Diabetes inpatient activity. 2007-8. Information Centre. http://ndis.ic.nhs.uk/pages/index.aspx.

3 National Diabetes Support Team (now NHS Diabetes). Improving emergency and inpatient care for people with diabetes 2008.

www.diabetes.nhs.uk/tools_and_resources/reports_and_guidance/
4 Diabetes UK. Collation of inpatient experiences. London: Diabetes UK, 2007. www.diabetes.org.uk/Documents/Professionals/ Surveys/Collation\%20of\%20Inpatient $\% 20$ Experiences $\% 20$ Final final.doc

5 NHS Diabetes. Safe use of insulin. www.diabetes.nhs.uk/ safe_use_of_insulin/

6 NHS Institute for Innovation and Improvement. Delivering quality and value: Focus on: Inpatient care for people with diabetes. London: NHSIII, 2008. www.institute.nhs.uk/quality_and_value/high_volume_ care/focus_on:_diabetes.html

7 Hillson R. Diabetes care: a practical manual. Oxford: Oxford University Press, 2008.

8 Diabetes UK. Putting feet first. Commissioning specialist services for management and prevention of diabetic foot disease in hospitals. London: Diabetes UK, 2009.

9 Griesdale DE, de Souza RJ, van Dam RM et al. Intensive insulin therapy and mortality among critically ill patients: a meta-analysis including NICE-SUGAR study data. CMAJ 2009;180:821-7.

10 American Diabetes Association. Standards of medical care in diabetes. Diabetes Care 2010;33(Suppl 1):S11-61v.

Address for correspondence: Dr R Hillson MBE, The Hillingdon Hospital, Uxbridge, Middlesex UB8 3NN. Email: rowan.hillson@thh.nhs.uk

\section{NCC-CC GUIDELINES}

\section{Type 2 Diabetes}

\section{National clinical guideline for management in primary and secondary care (update)}

Type 2 diabetes can cause severe complications, affecting the eye, the nervous system and the kidney. The overall risk of cardiovascular disease is more than doubled, and life expectancy is reduced by an average seven years. In 2002, the National Institute for Health and Clinical Excellence (NICE) published a suite of five guidelines dealing with different aspects of the care of type 2 diabetes. The rising prevalence of the disease, and the range of complications which can arise, reinforce the importance of up-todate guidance and accordingly NICE have asked the NCC-CC to produce this guideline, amalgamating and updating the previously published work.
Topics of particular relevance to life expectancy, such as control of cholesterol and lipid levels, and management of hypertension, are covered in the guideline. It deals with major complications such as renal disease. There are also key recommendations in areas of great importance to patients such as structured education and the monitoring of glucose levels. Naturally, there are also sections dealing with control of blood glucose levels and the use of the various drugs available for this purpose.

The guideline is an invaluable resource for general physicians, diabetologists, dieticians, general practitioners, nurses and healthcare professionals who are involved in the management and care of people with type 2 diabetes.

Developed by the National Collaborating Centre for Chronic Conditions at the Royal College of Physicians

\section{Published May 2008 ISBN 9781860163333}

Price: $£ 33.00$ UK, $£ 36.00$ overseas (prices include postage and packing) $10 \%$ discount on all RCP publications to fellows and members

Please quote the reference Clinical Medicine when making your order 